\title{
Numerical simulation of arterial remodeling in pulmonary autografts.
}

\author{
Nele Famaey $^{a *}$, Julie Vastmans $^{a *}$, Heleen Fehervary ${ }^{a *}$, Lauranne Maes $^{a *}$, \\ Emma Vanderveken $^{b *}$, Filip Rega ${ }^{b *}$, S. Jamaleddin Mousavi ${ }^{d *}$, Stéphane \\ Avril $^{d *}$ \\ ${ }^{a}$ Biomechanics Section, Mechanical Engineering Department, KU Leuven, Leuven, Belgium \\ ${ }^{b}$ Clinical Cardiac Surgery, Department of Cardiovascular Sciences, KU Leuven, Leuven, \\ Belgium \\ ${ }^{d}$ Mines Saint-Etienne, Univ Lyon, Univ Jean Monnet, INSERM, U 1059 Sainbiose, Centre \\ CIS, F - 42023 Saint-Etienne France
}

\begin{abstract}
The Ross procedure is a surgical procedure where a diseased aortic valve is replaced by the person's own pulmonary valve. The proximal segment of the pulmonary artery is thereby placed in aortic position and therefore suddenly exposed to a sevenfold increase in blood pressure. Excessive dilatation of this pulmonary autograft is a common complication and has excited interest about mechanobiological adaptation in such situations. Mathematical modelling of growth and remodelling is a relevant approach to improve insights into this phenomenon.

We introduced an algorithm that models the continuous degradation and deposition of extracellular matrix in an artery according to the constrained mixture theory. To compute temporal variations of collagen and elastin mass and to deduce related mechanical properties of the remodelled artery, we discretized time and defined a finite number of cohorts for each collagen fiber family. The degradation and production rates of each cohort were mediated by the difference between ambient and homeostatic stress within each fiber cohort.
\end{abstract}

*Corresponding author: N. Famaey, KU Leuven, Biomechanics Section, Celestijnenlaan 300C, 3001 Heverlee (Belgium)

Email address: nele.famaey@kuleuven.be (Stéphane Avril ${ }^{d}$ )

Preprint submitted to Journal of Applied Mathematics and Mechanics

June 8, 2018 
We applied the algorithm to predict the adaptation of a pulmonary autograft over an extended period and compared the results to experimental data obtained in sheep. We were able to consistently reproduce the experimentally observed remodeling effects such as dilatation and delayed collagen fiber recruitment. Our simulations revealed how elastin takes up the excessive stress in overstretched regions of the tissue.

In conclusion, the algorithm yields very promising results regarding autograft adaptation in overstretched conditions. Future work will focus on other situations of vascular adaptation where growth-related deformations will be considered.

Keywords: growth \& remodeling, arteries, constrained mixture theory, pulmonary autograft

\section{List of Symbols}

$A_{a}^{e x p} \quad$ Stress-stretch curves of planar biaxial tests of healthy aortic tissue

$A F S(s) \quad$ Average stress in a fiber family at time $s$, see equation 23

a $\quad$ Undeformed collagen fiber orientation vector

$\overline{\mathbf{C}}^{\alpha} \quad$ Deviatoric part of a constituent-specific right Cauchy-Green tensor, see equation 3

$C C S(s, \tau)$ Stress in a certain cohort $\tau$ at the current time $s$, see equation 20

DCS Stress in a cohort at the time of its deposition, see equation 21

$d_{i} \quad$ Vessel diameter in pulmonary $(i=P)$ and aortic $(i=A)$ conditions, see Table 2

$\mathbf{F}(s) \quad$ Global deformation gradient tensor at time $s$, see equation 5

$\overline{\mathbf{F}}(s) \quad$ Global deviatoric deformation gradient tensor at time $s$, see equation 4 


\begin{tabular}{|c|c|}
\hline$\overline{\mathbf{F}}^{\alpha}$ & Constituent-specific deformation gradient tensor, see equation 4 \\
\hline $\mathbf{G}^{\alpha}$ & $\begin{array}{l}\text { Constituent-specific deposition stretch tensor, see equations } 13 \text { and } \\
14\end{array}$ \\
\hline$g^{\text {coll }}$ & Collagen fiber deposition stretch value, see equation 13 \\
\hline$g_{a x}^{\text {elas }}$ & Axial elastin deposition stretch value \\
\hline$g_{\text {circ }}^{\text {elas }}$ & Circumferential elastin deposition stretch value \\
\hline $\mathbf{I}$ & Identity tensor \\
\hline $\bar{I}_{1}^{\text {elas }}$ & $\begin{array}{l}\text { First invariant of the deviatoric right Cauchy-Green tensor of the } \\
\text { elastin constituent }\end{array}$ \\
\hline $\bar{I}_{4}^{\alpha}$ & $\begin{array}{l}\text { Fourth invariant of the deviatoric right Cauchy Green tensor of a } \\
\text { fiber cohort }\end{array}$ \\
\hline$J$ & $\begin{array}{l}\text { Jacobian, i.e. determinant of the deformation gradient tensor, see } \\
\text { equation } 5\end{array}$ \\
\hline$K_{\sigma}^{\alpha}$ & Rate parameter, see equation 7 \\
\hline$K_{q_{h}}^{\alpha}$ & $\begin{array}{l}\text { Homeostatic decay constant and basal production rate for constituent } \\
\alpha \text {, see equations } 6,9 \text { and } 27\end{array}$ \\
\hline$\hat{K}_{q}^{\alpha}(s, \tau)$ & Decay constant at time $s$ for a cohort $\tau$, see equations 915 and 27 \\
\hline$k$ & Current increment \\
\hline$l_{i 0}$ & $\begin{array}{l}\text { Length of the surface perpendicular to the } i \text {-th direction at the start } \\
\text { of the virtual biaxial test }\end{array}$ \\
\hline$m^{\alpha}(0)$ & Homeostatic rate of production of constituent $\alpha$, see equation 6 \\
\hline$m^{\alpha}(\tau)$ & $\begin{array}{l}\text { Rate of production of constituent } \alpha \text { at time } \tau \in[0, s] \text {, see equations } \\
7 \text { and } 18\end{array}$ \\
\hline
\end{tabular}


$p_{i} \quad$ Blood pressure in pulmonary $(i=P)$ and aortic $(i=A)$ conditions, see Table 2

$P_{p}^{m o d} \quad$ Results of test 1 , i.e. a virtual biaxial test after finding deposition stretches under pulmonary loading conditions

$P_{p r}^{m o d} \quad$ Results of test 2, i.e. a virtual biaxial test after remodeling under pulmonary loading conditions

$P_{a r_{-} \sigma}^{\bmod } \quad$ Results of test 3, i.e. a virtual biaxial test after remodeling under aortic loading conditions

$P_{a r_{-} \text {noo }}^{\text {mod }} \quad$ Results of test 4, i.e. a virtual biaxial test after remodeling under aortic loading conditions, without stress-dependencies

$P_{p}^{e x p} \quad$ Stress-stretch curves of planar biaxial tests of healthy pulmonary artery

$P_{a}^{e x p} \quad$ Stress-stretch curves of planar biaxial tests of pulmonary autograft after 6 months of implantation

$Q^{\alpha}(s) \quad$ Fraction of constituent $\alpha$ at time $s=0$ that survives to time $s$, see equation 2 and 26

$q^{\alpha}(s, \tau) \quad$ Fraction of constituent $\alpha$ that was produced at $\tau$ that survives to $s$, see equation 8

$R F_{i} \quad$ Resulting force in the i-direction during the virtual biaxial test

$s \quad$ Current time

$t_{i 0} \quad$ Thickness of the surface perpendicular to the $i$-th direction at the start of the biaxial test

$t_{i} \quad$ Vessel wall thickness in pulmonary $(i=P)$ and aortic $(i=A)$ conditions, see Table 2

$x_{M} \quad$ X-coordinate of marker 


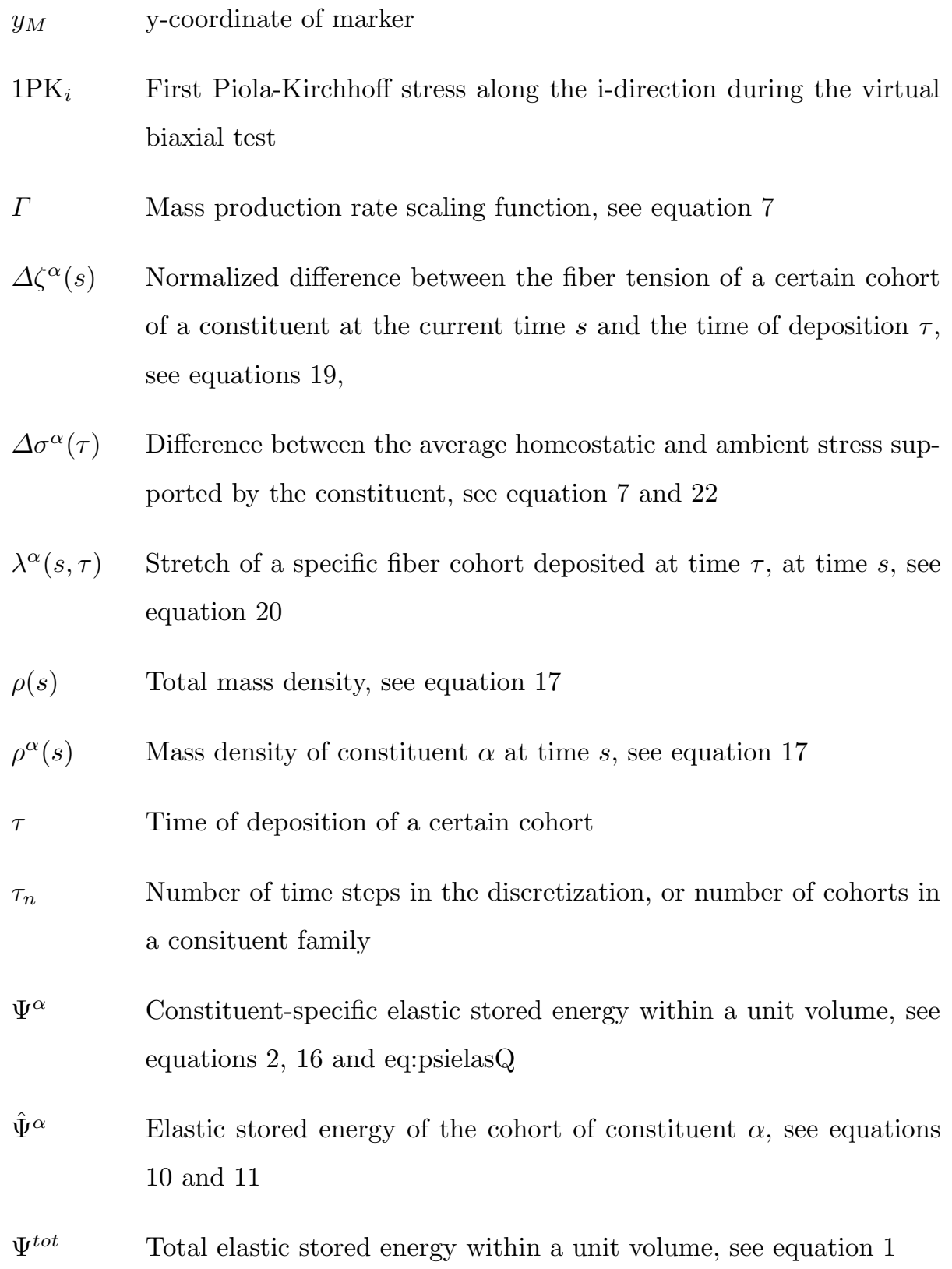

\section{Introduction}

Mechanical stresses dictate, at least in part, the way in which the microstructure of soft tissues is organized. This observation has led to the concept of 
functional adaptation wherein it is thought that soft tissues functionally adapt so as to maintain particular mechanical metrics (e.g. stress) near target values. To accomplish this, tissues often develop regionally varying stiffness, strength and anisotropy [1].

A particular situation of such adaptation in cardiac surgery is the Ross procedure 22. For a patient with a dysfunctional aortic valve, it consists in replacing the damaged aortic valve with the native pulmonary valve, which will often function well and can grow if the patient is a child. The pulmonary valve itself has to be replaced with a graft valve. For practical reasons, the proximal part of the aorta (aortic root) is also replaced by the proximal segment of the pulmonary artery (pulmonary root) during the Ross procedure.

The systemic blood pressure is on average seven times larger than the pulmonary pressure, and the new mechanical conditions applied on the pulmonary autograft are the source of adaptation of the tissue. Dilatation of the pulmonary autograft after the Ross operation in adults has even become a matter of serious concern 3, 4. Disproportionate enlargement of the neoaortic root has been documented in children after the Ross operation [5] as well as after the arterial switch operation, and has been related to neoaortic valve regurgitation [6].

These observations have excited the interest about how mechanobiological adaptation of the pulmonary root to systemic pressures is accomplished. Various groups have observed that vascular remodeling due to increased mechanical stimulus is primarily characterized by an increased matrix deposition that results in a thicker wall [7, 8, 9, 1]. This mode of remodeling, to maintain wall integrity and restore baseline conditions of wall stress, is well known in systemic arteries, pulmonary arteries, and veins subject to hypertension.

As described in [10], we preliminarily characterized the adaptive biomechanical responses in vascular wall explants of mature sheep in which, six months before the characterization, a valveless pulmonary autograft had been implanted in the aorta. Our results show that a valveless pulmonary autograft placed in aortic position will remodel such that its macroscopic behavior leans more towards aorta-like behavior, as measured through planar biaxial tensile tests. In 
contrast, when studying excessively dilated pulmonary autografts in ten patients, Mookhoek et al. [11, 12] observed a decreased stiffness compared to both the native aorta and pulmonary artery, suggesting a failure of the tissue to remodel.

Developing a mathematical model about this phenomenon may help to understand neoaortic valve failures, assuming that pulmonary (neoaortic) root dilatation is a major determinant of neoaortic valve regurgitation. Therefore, the aim of our study is to mathematically describe remodeling of the pulmonary arterial wall. To predict such biomechanical adaptation we invoke the theory of growth and remodeling which embeds the reaction-diffusion framework within tissue mechanics [13].

The mathematical framework of growth and remodeling dates back to [14, 15. The primary assumption is that one models volumetric growth through a growth tensor $\mathbf{F}_{g}$, which describes changes between two fictitious stress-free configurations: the original body is imagined to be fictitiously cut into small stress-free pieces, each of which is allowed to grow separately via $\mathbf{F}_{g}$. Because these growths need not be compatible, internal forces are often needed to assemble the grown pieces, via $\mathbf{F}_{a}$, into a continuous configuration. This, in general, produces residual stresses, which are now known to exist in many soft tissues. The formulation is completed by considering elastic deformations, via $\mathbf{F}_{e}$, from the intact but residually stressed traction-free configuration to a current configuration that is induced by external mechanical loads. The initial boundary value problem is solved by introducing a constitutive relation for the stress response to the deformation $\mathbf{F}_{e} \mathbf{F}_{a}$, which is often assumed to be incompressible hyperelastic, plus a relation for the evolution of the stress-free configuration via $\mathbf{F}_{g}$.

Although the previous theory, called the theory of kinematic growth, yields many reasonable predictions, Humphrey and coworkers [13] have suggested that it models consequences of growth and remodeling, not the processes by which they occur. Growth and remodeling necessarily occur in stressed, not fictitious stress-free, configurations, and they occur via the production, removal, and or- 
ganization of different constituents; moreover, growth and remodeling need not restore stresses exactly to homeostatic values. Hence, Humphrey and coworkers introduced a conceptually different approach to model growth and remodeling, one that is based on tracking the turnover of individual constituents in stressed configurations (the constrained mixture model [13]). A comprehensive review comparing both approaches is provided in Cyron et al. [16].

We have chosen to model the adaptation of pulmonary autografts using constrained mixture theory. The main advantage of this mathematical framework compared to kinematic growth is that it takes into account the simultaneous very different production and degradation processes that both elastin and collagen have during the adaptation process. The other advantage is that it allows to consider the different in situ stresses experienced by collagen and elastin in the artery. In situ stresses are well evidenced, for instance when arteries are excised during a surgical intervention. Due to the cancellation of in vivo loadings, the artery experiences an elastic recoiling. Actually, the elastic recoiling manifests mostly by recoiling of elastic fibers, whereas collagen fibers go from a straight state to a crimped state where they bear no more tensile stress.

To incorporate these processes, so-called constrained mixture models have already been established by a number of authors [17, 18, 19, 20, 21, 22, 23, 24, 25], including homogenized approaches [26, 27]. They describe growth and remodeling as a superposition of continuous degradation and deposition processes of differential mass increments of multiple constituents in each differential volume element. Each constituent has a given deposition stretch in the configuration at which it is created.

In the present paper, we introduce an original algorithm implemented in the Abaqus finite-element software to model the continuous degradation and deposition processes of collagen and elastin in an artery according to the constrained mixture theory. After presenting in detail this algorithm, we show its very good ability to predict the biomechanical adaptation of pulmonary autografts by comparing the predictions to our experimental results in the sheep experiments [10]. 


\section{Materials and Methods}

\subsection{Constrained-mixture remodeling theory}

We choose to model the artery according to the constrained mixture theory as previously described in [13. Within this mixture and for the specific application of this study, we consider the mechanical contribution of an elastin matrix and two fiber families of collagen. These can be modelled as a composite material made of a matrix, representing the contribution of elastin, reinforced by two fiber families, representing the contribution of collagen. The fiber families are oriented in a plane perpendicular to the radial axis, at angles symmetric with respect to the circumferential direction.

In 20, the total elastic stored energy $\Psi^{\text {tot }}$ within a unit volume of material containing these constituents is calculated using a mass-averaged rule

$$
\Psi^{\mathrm{tot}}=\sum_{\alpha} \Psi^{\alpha}
$$

with

$$
\Psi^{\alpha}=\frac{\rho^{\alpha}(0)}{\rho(s)} Q^{\alpha}(s) \hat{\Psi}^{\alpha}\left(\overline{\mathbf{C}}_{n(0)}^{\alpha}(s)\right)+\int_{0}^{s} \frac{m^{\alpha}(\tau)}{\rho(s)} q^{\alpha}(s, \tau) \hat{\Psi}^{\alpha}\left(\overline{\mathbf{C}}_{n(\tau)}^{\alpha}(s)\right) d \tau,
$$

where $\alpha$ denotes the constituent type (elastin, collagen fiber family 1 or collagen fiber family 2) and where

- $\rho^{\alpha}(0)$ is the mass density of constituent $\alpha$ at time $s=0$, i.e. when remodeling starts,

- $\rho(s)$ is the total mass density,

- $Q^{\alpha}(s)$ is the fraction of constituent $\alpha$ at time $s=0$ that survives to time $s$,

- $m^{\alpha}(\tau)$ is the rate of production of constituent $\alpha$ at time $\tau \in[0, s]$,

- $q^{\alpha}(s, \tau)$ is the fraction of constituent $\alpha$ that was produced at $\tau$ that survives to time $s$ and 
- $\hat{\Psi}^{\alpha}$ is the elastic stored energy of the cohort of constituent $\alpha$.

All variables are also described in the list of symbols section.

Here, $\hat{\Psi}^{\alpha}$ depends on the individual deviatoric right Cauchy-Green tensor of each cohort of each constituent,

$$
\overline{\mathbf{C}}^{\alpha}=\overline{\mathbf{F}}^{\alpha T} \overline{\mathbf{F}}^{\alpha}
$$

with $\overline{\mathbf{F}}^{\alpha}$ the constituent-specific deviatoric deformation gradient. The latter differs per cohort and per constituent for two reasons. Firstly, each constituent type can have a specific degree of stretch with which it is deposited into the tissue, expressed by the deposition stretch tensor $\mathbf{G}^{\alpha}$. Secondly, each cohort of a constituent is deposited at a different time point $\tau$ and will hence only start to deform along with the mixture from that time point on. This yields as total constituent-specific deformation gradient at time $s$

$$
\overline{\mathbf{F}}^{\alpha}=\overline{\mathbf{F}}(s) \overline{\mathbf{F}}^{-1}(\tau) \mathbf{G}^{\alpha},
$$

with $\overline{\mathbf{F}}(s)$ the global deviatoric deformation gradient at the current time $s$ and $\overline{\mathbf{F}}^{-1}(\tau)$ the inverse of the global deviatoric deformation gradient at the time of deposition of the constituent cohort $\tau$. Note that only the deviatoric part of the deformation gradient is considered here,

$$
\overline{\mathbf{F}}=J^{-1 / 3} \mathbf{F}
$$

with $J$ the Jacobian or determinant of $\mathbf{F}$. Please refer to the discussion section for a discussion on volumetric growth.

In a matured artery, the rate of production of elastin is assumed to be zero [13. Collagen production is assumed to have a positive basal rate during homeostasis

$$
m^{\alpha}(0)=\rho^{\alpha}(0) K_{q_{h}}^{\alpha}
$$


which can be influenced by the ambient (i.e. current) stress level, as described in equations (48-50) in [20]. Here, $K_{q_{h}}^{\alpha}$ is the basal production rate for constituent $\alpha$. In our implementation, we neglect the effect of wall shear stress, yielding

$$
m^{\alpha}(\tau)=m_{0}^{\alpha} \Gamma\left(K_{\sigma}^{\alpha}, \Delta \sigma^{\alpha}(\tau)\right),
$$

where $K_{\sigma}^{\alpha}$ is a rate parameter and $\Delta \sigma^{\alpha}(\tau)$ quantifies the difference between the average homeostatic and ambient stress supported by the collagen fiber family. $\Gamma$ is a mass production rate scaling function, that basically sets a maximum mass density production value and relates the stress difference and production rate.

To compute temporal variations of each constituent's mass and mechanical properties, we discretized time in different time steps and introduced the concept of cohorts for each fiber family, a cohort being the mass of this constituent produced at a given time step. We assume that the mass of each cohort decays exponentially during the time steps following its production, at a degradation rate $q^{\alpha}$ which is again influenced by the difference between ambient and homeostatic stress within the constituent,

$$
q^{\alpha}(s, \tau)=\exp \left(-\int_{\tau}^{s} \hat{K}_{q}^{\alpha}(s, \tilde{\tau}) d \tilde{\tau}\right)
$$

with,

$$
\hat{K}_{q}^{\alpha}(s, \tau)=K_{q_{h}}^{\alpha}\left(1+\Delta \zeta^{\alpha 2}\right),
$$

with $\Delta \zeta^{\alpha}(s)$ the normalized difference between the fiber tension of a certain cohort of a collagen fiber family at the current time $s$ and the time of deposition $\tau$, and $K_{q h}^{\alpha}$ the homeostatic constant of decay for collagen, also used to describe the basal production rate (see equation 7 ). The above three equations can also be found in equations (50-53) of [20].

Returning to equation 2, formulations for the strain energy function of each constituent are still to be defined. For elastin, a basic neo-Hookean formulation 
is used,

$$
\hat{\Psi}^{\text {elas }}=C_{10}^{m}\left(\bar{I}_{1}^{\text {elas }}-3\right),
$$

where $\bar{I}_{1}^{\text {elas }}$ is the first invariant of the deviatoric right Cauchy-Green tensor of the elastin constituent and $C_{10}^{m}$ is the shear modulus per unit mass.

For collagen, an exponential strain energy function is used [28, yielding for each cohort

$$
\hat{\Psi}^{\alpha}=\frac{k_{1}^{m}}{2 k_{2}}\left(\exp \left[k_{2}\left(\bar{I}_{4}^{\alpha *}-1\right)^{2}\right]-1\right),
$$

and

$$
\bar{I}_{4}^{\alpha *}=\kappa \bar{I}_{1}^{\alpha}+(1-3 \kappa) \bar{I}_{4}^{\alpha} .
$$

where, $\bar{I}_{4}^{\alpha}=\mathbf{a}_{\mathbf{0}} \cdot \overline{\mathbf{C}}^{\alpha} \mathbf{a}_{\mathbf{0}}$ is the fourth invariant of the deviatoric right Cauchy

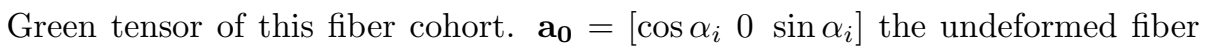
orientation vector. $\alpha_{i}$ is the angle of the fibers with respect to the circumferential direction of the artery. $k_{1}^{m}$ is a stress-like parameter describing the fiber stiffness, again per unit mass, and $k_{2}$ is a dimensionless parameter related to the stiffening of the collagen fibers at higher pressures. $\kappa$ incorporates the dispersion of the fibers around the main orientation described with $\alpha_{i} . \kappa$ is a positive number between 0 (no dispersion) and $1 / 3$ (isotropic dispersion). The implementation is such that no fiber contribution is considered when the fibers are (on average) under compression, i.e. when $\bar{I}_{4}^{\alpha *}<1$.

\subsection{Numerical implementation}

The constrained mixture remodeling algorithm was numerically implemented in a user-defined subroutine (UMAT) in Abaqus 6.14 (Simulia, Dassault-Systemes). This fortran-based subroutine is called for each integration point in each iteration of an implicit finite element scheme, and serves to compute the Cauchy stress tensor at this integration point, as well as the consistent tangent stiffness tensor. To compute these tensors, several variables are passed into the 
subroutine for information, including a.o. the current deformation gradient $\mathbf{F}$ and material parameters as defined in the input file. A number of solutiondependent variables can also be updated throughout the subroutine and are passed on to each new increment. Below, we introduce the algorithm defining deposition stretches before describing the remodeling algorithm.

\subsubsection{Finding deposition stretches}

As mentioned in equation 4, the elastin and collagen constituents of an artery are prestretched in their homeostatic reference configuration, with deposition stretch tensors $\mathbf{G}^{\text {elas }}$ and $\mathbf{G}^{\text {coll }}$, respectively. For the collagen deposition stretches, $\mathbf{G}^{\text {coll }}$ is defined such that a stretch value of $g^{\text {coll }}$ is obtained along the collagen fiber direction, as [26]:

$$
\mathbf{G}^{\text {coll }}=g^{\text {coll }} \mathbf{a}_{\mathbf{0}} \otimes \mathbf{a}_{\mathbf{0}}+\frac{1}{\sqrt{g^{\text {coll }}}}\left(\mathbf{I}-\mathbf{a}_{\mathbf{0}} \otimes \mathbf{a}_{\mathbf{0}}\right),
$$

with $\mathbf{I}$ the identity tensor and $\mathbf{a}_{\mathbf{0}}$ the undeformed collagen fiber orientation vector. We assume $g^{\text {coll }}$ to be a known constant for all fibers in the geometry, as previously done by [17] and [29].

In contrast, elastin is very stable, with a half-life of over 40 years, implying that it has deformed during the subject's growth. As a consequence, $\mathbf{G}^{\text {elas }}$ will vary from material point to material point, leading to a considerable amount of unknowns. However, for a known set of material parameters and assuming a constant axial stretch value $g_{a x}^{\text {elas }}$ and isochoric deformation, the solution to this problem is unique. Effectively, the deposition stretches serve to balance the reference configuration, in casu the configuration in the pulmonary artery, with the loads present in this configuration, in casu pulmonary pressure.

Hence the unknown values $g_{\text {circ }}^{\text {elas }}$ can be obtained through an iterative scheme similar to [17]: an FE simulation of the artery is performed, in which the pulmonary geometry is meshed and initially load- and stress-free. In the first step of the analysis, the stress-free geometry is deformed by the pulmonary pressure. In the following step, the collagen deposition stretch $g^{\text {coll }}$ and an elastin 
deposition stretch $\mathbf{G}^{\text {elas }}$ is assigned to each material point as

$$
\mathbf{G}^{\text {elas }}=\left[\begin{array}{ccc}
\frac{1}{\sqrt{g_{a x}^{\text {elas }}}} & 0 & 0 \\
0 & \frac{1}{\sqrt{g_{a x}^{\text {elas }}}} & 0 \\
0 & 0 & g_{a x}^{\text {elas }}
\end{array}\right] .
$$

This induces material stiffening and partial recoiling towards the reference configuration. Next, the resulting deformation relative to the reference configuration is also assigned to elastin by multiplying it with the already existing deposition stretch tensor at each material point. This again induces stiffening and recoiling. If at the end of this step the reference configuration has not been fully recovered yet, the remaining deformation will be multiplied to the already existing elastin deposition stretch. This iterative loop is repeated until the final configuration matches the reference configuration up to a desired level of accuracy, i.e. until the magnitude of the average nodal displacement is below a threshold value $u_{t}$. A suggestion for $u_{t}$ is $1 \%$ of the diastolic wall thickness. Due to implementation practicalities in Abaqus, the iterative loop is repeated a finite number of times, higher than the amount required to reach the threshold value. A schematic of this workflow can be found in Figure 1 .

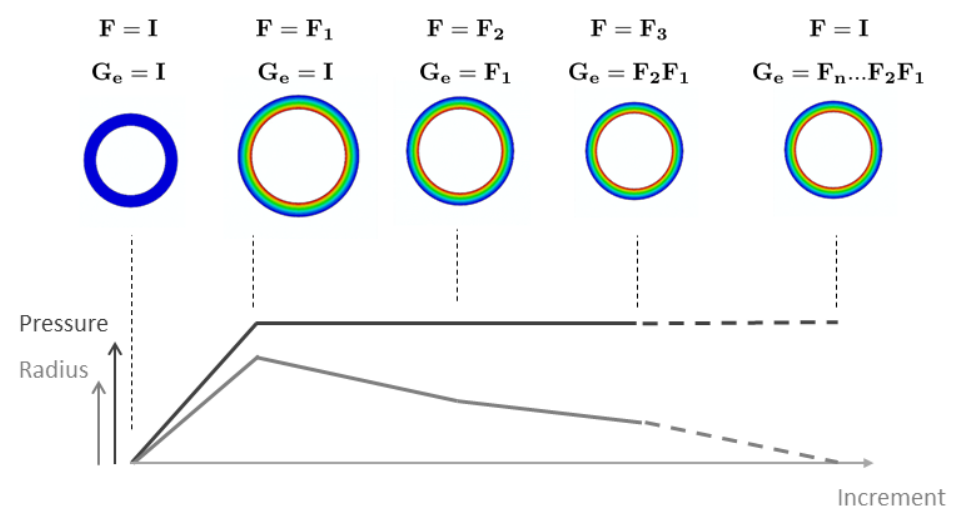

Figure 1: Schematic representation of the algorithm to find the deposition stretches of elastin. 


\subsubsection{Remodeling algorithm}

After finding the deposition stretches, the homeostatic reference configuration is known and simulations of remodeling can be started. At each time step, a converged solution is sought, where the rate of production $m^{\alpha}(\tau)$ and rate of degradation $q^{\alpha}(s, \tau)$ of each cohort matches with the current stress state. This is achieved through direct time stepping (using the keyword *Static, direct), where each increment $(k)$ serves as intermediate time steps in the convergence process. Note that within each increment, several iterations $(i)$ might also be required to solve the finite element equilibrium.

As described in equation 2, after initializing mass fractions of each constituent, elastin mass can only degrade. Collagen on the other hand, is continuously deposited and degraded, and its total contribution is computed through integration. Due to our simulation design with the concept of cohorts of collagen fibers, integration is easily achieved as a discrete sum. Furthermore, after a finite amount of time steps $\tau_{n}$, due to the degradation rate, the contribution of cohorts older than $\tau_{n}$ time steps will have a negligible contribution and can be removed. Consequently, we can allocate a finite number of slots $\tau_{n}$ for collagen cohorts within each fiber family. This concept is schematically represented in Figure 2 ,

For each collagen cohort of a fiber family $\alpha=$ coll deposited at time $\tau=$ $\left[1, \tau_{n}\right]$ we store,

- the inverse of the total deformation gradient at the time the cohort was deposited $\mathbf{F}^{-1}(\tau)$,

- the mass fraction of collagen that was initially deposited at time $\tau, m^{\alpha}(\tau)$, as in equation 7

- the decay constant of the survived fraction of this cohort at the current time increment $s=k$, and at the previous time increment $s=k-1$, i.e

$$
\hat{K}_{q}^{\alpha}(k, \tau)=\hat{K}_{q}^{\alpha}(k-1, \tau)-K_{q_{h}}^{\alpha}\left(1+\Delta \zeta^{2}\right),
$$




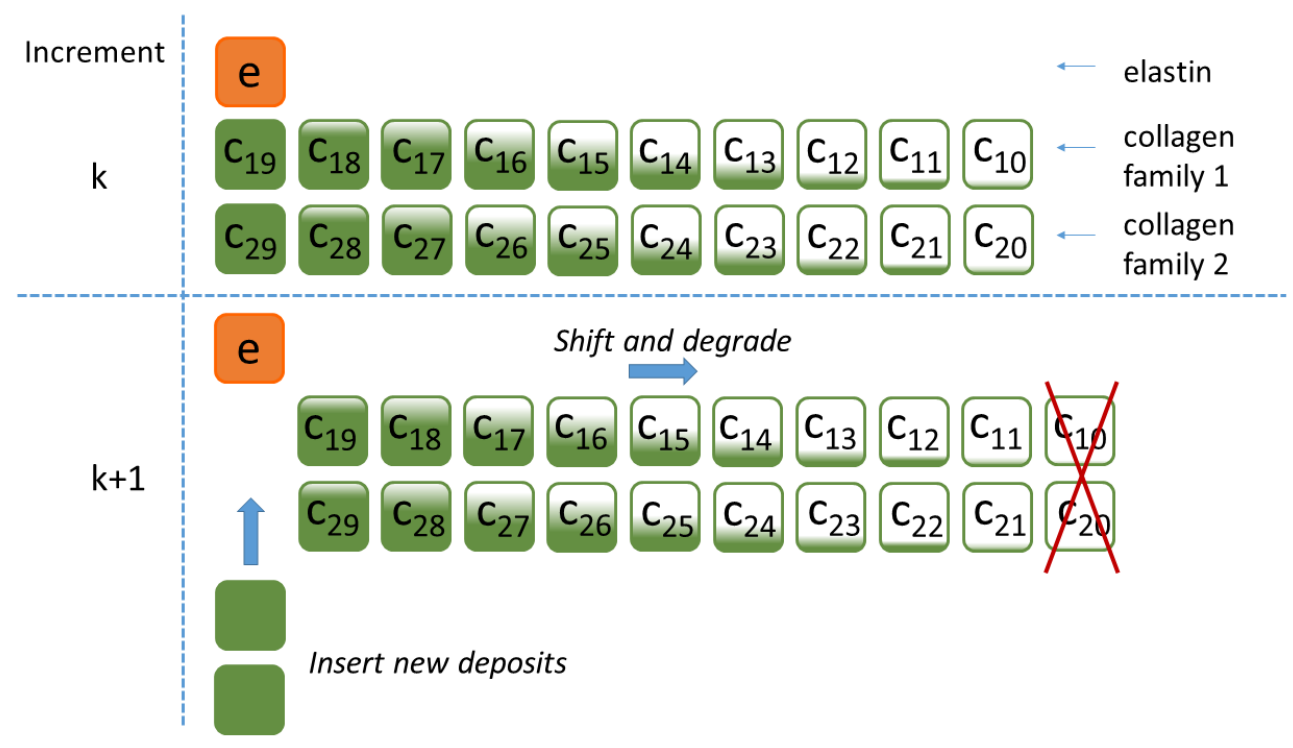

Figure 2: Schematic representation of the remodeling algorithm. There is one cohort of elastin (e) that is only allowed to degrade. For each collagen fiber family, there are $\tau_{n}=10$ cohorts. Each cohort was deposited at a different time step. The leftmost cohort was deposited at the current time point and has therefore not been degraded yet. The rightmost cohort was deposited $\tau_{n}$ time steps before the current time step and has been almost fully degraded. At the next time step, this final cohort will be discarded and a new one will be added. 
which is the discretized version of equation 9 . This way, equation 2 for a collagen family $\alpha$ in a certain increment $k$ simplifies to

$$
\Psi^{\alpha}=\sum_{\tau=1}^{\tau_{n}} \frac{m^{\alpha}(\tau)}{\rho(k)} q^{\alpha}(k, \tau) \hat{\Psi}^{\alpha}\left(\overline{\mathbf{C}}_{\tau}^{\alpha}\right)
$$

where

$$
\rho(k)=\rho^{\text {elas }}(0)+\sum_{\tau=1}^{\tau_{n}} \rho^{\alpha}(\tau),
$$

with

$$
\rho^{\alpha}(\tau)=m^{\alpha}(\tau)_{k} q^{\alpha}(k, \tau)
$$

To derive the survival rate $\Delta \zeta$ of equation 9 , we compute the stress in each cohort at the current increment $(C C S(s, \tau))$ and compare it to the stress of this cohort at the time of its deposition $(D C S)$. This yields

$$
\Delta \zeta^{\alpha}(k)=\frac{C C S}{D C S}-1
$$

with

$$
\begin{aligned}
C C S(k, \tau) & =\frac{\partial \hat{\Psi}^{\alpha}}{\partial \lambda^{\alpha}}\left(\lambda^{\alpha}(k, \tau)\right), \\
D C S & =\frac{\partial \hat{\Psi}^{\alpha}}{\partial \lambda^{\alpha}}\left(g^{\text {coll }}\right) .
\end{aligned}
$$

Here, $\lambda^{\alpha}$ is the stretch in the fiber direction and $\lambda^{\alpha}(k, \tau)$ the total stretch of the specific cohort deposited at time $\tau$ at increment $k$.

To derive the production rate, we can take a closer look at equation 7 and apply it to the current algorithm. $\Delta \sigma^{\alpha}(s)$ is computed as the difference between the average collagen fiber stress at homeostasis $A F S(0)$ and the average fiber stress at time $s, A F S(s)$.

$$
\Delta \sigma^{\alpha}(k)=A F S(k)-A F S(0) .
$$


This average fiber stress is weighted according to the current mass fraction of each cohort which, for a certain time increment $k$ yields

$$
A F S(k)=\sum_{i=1}^{\tau_{n}} \frac{m^{\alpha}(\tau)}{\rho(k)} q^{\alpha}(k, \tau) \frac{\partial \hat{\Psi}^{\alpha}}{\partial \lambda^{\alpha}}\left(\lambda^{\alpha}(k, \tau)\right) .
$$

As elastin is only allowed to degrade, its contribution to the total strain energy density at increment $k$ of this discretized implementation simplifies to

$$
\Psi^{\text {elas }}=\frac{\rho^{\text {elas }}(0)}{\rho(k)} Q^{\text {elas }}(k) \hat{\Psi}^{\text {elas }}\left(\overline{\mathbf{C}}^{\text {elas }}\right),
$$

with

$$
\begin{aligned}
\overline{\mathbf{C}}^{\text {elas }} & =\overline{\mathbf{F}}^{\text {elasT }} \overline{\mathbf{F}}^{\text {elas }}, \text { with } \overline{\mathbf{F}}^{\text {elas }}=\overline{\mathbf{F}} \mathbf{G}^{\text {elas }}, \\
Q^{\text {elas }}(k) & =\exp \left(\hat{K}_{q}^{\text {elas }}(k, 0)\right), \text { with } \\
\hat{K}_{q}^{\text {elas }}(k, 0) & =\hat{K}_{q}^{\text {elas }}(k-1,0)-K_{q_{h}}^{\text {elas }}\left(1+\Delta \zeta^{\text {elas }}(k)^{2}\right) .
\end{aligned}
$$

Using expressions 10 and 11, the contributions to the Cauchy stress tensor and the consistent tangent stiffness tensor can be derived as described in the Appendix of [30.

Note that, as mentioned in Table 1 we use a homeostatic decay constant for collagen $K_{q_{h}}^{\text {coll }}=\log 2.0 / 70$ days $^{-1}$. This means that after 70 days, half of a given collagen cohort has disappeared. As can also be seen in Figure 2, the current implementation allows a cohort of collagen to degrade in $\tau_{n}$ discrete time steps. In order to have a negligibly small percentage of collagen left after $\tau_{n}$ time steps, $\tau_{n}$ should be set high enough. On the other hand, $\tau_{n}$ should not be too high for computational reasons. We found a trade-off with $\tau_{n}=10$, which means that the time step in Abaqus corresponds to 70 days. However, to investigate the effect of $\tau_{n}$, we also repeated the simulations for varying levels of $\tau_{n}=[5,10,20,30]$.

In the current implementation, and as discussed in section 4 , we do not explicitly account for volumetric growth. Instead, to account for the influence of extra constituent deposition, we modify equation 2 by changing $\rho(s)$ to $\rho(0)$. 
This means that the fraction of each constituent is calculated relatively to the initial density rather than to the current.

\subsection{Application in the simplified ROSS procedure}

\subsubsection{Experimental protocol}

As a test case for the algorithm described in the previous section, we use the results obtained by [10] and [31. In this experimental study, a section of the pulmonary autograft was placed in the descending aorta in two sheep. This procedure serves as a simplified version of the Ross procedure described in the introduction. Six months after the procedure, the sheep were sacrificed and planar biaxial tests were carried out on the following tissues: healthy pulmonary artery, healthy aorta, and pulmonary artery (autograft) that remained in the aortic position for six months. A more detailed description of the experimental protocol can be found in [10].

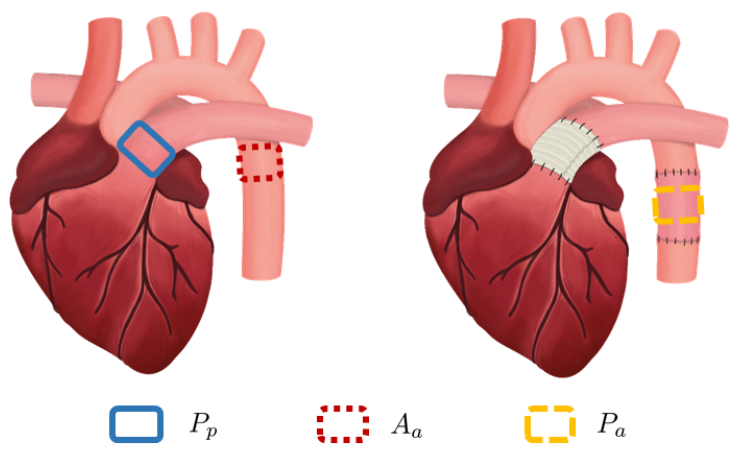

Figure 3: Schematic representation of the simplified Ross procedure, after which three relevant tissues are harvested: healthy pulmonary artery $\left(P_{p}\right)$, healthy aorta $\left(A_{a}\right)$, and pulmonary artery (autograft) that that remained in the aortic position for six months $\left(P_{a}\right)$. Courtesy of Brecht Vanderveken.

\subsubsection{Numerical workflow}

The aim of this study is to simulate the remodeling process of a pulmonary artery after placing it under aortic pressure. To evaluate the simulation results, the stress-strain behavior of the virtual tissue is tested through a virtual biaxial 
test, before and after remodeling. As such, three stages are identified within the remodeling scheme: the deposition stage, the remodeling stage and the tensile testing stage.

In the deposition stage, no remodeling is allowed to take place, i.e. the mass fractions of each constituent remain constant. In this stage, the deposition stretch tensor of the elastin constituent $\mathbf{G}^{\text {elas }}$ is found for each integration point using the iterative scheme described in section 2.2.1.

In the remodeling stage, remodeling takes place as described in section 2.2 .2 In the tensile testing stage, no remodeling takes place, but the total Cauchy stress and consistent tangent stiffness matrix are calculated using the configuration (w.r.t. densities and constituent-specific deformation gradients) of the final step of the remodeling stage.

A single linear hexahedral element $(\mathrm{C} 3 \mathrm{D} 8 \mathrm{H})$ is used with Abaqus hybrid formulation to prescribe incompressiblity. No volume changes are considered (see discussion). Table 1 reports the values of material parameters. Depending on the algorithm stage, different boundary conditions are assigned. Figure 4 shows the element and indicates the different faces upon which boundary conditions are applied. In the image, the $\mathrm{X}$-axis corresponds to the circumferential direction, the $\mathrm{Y}$-axis to the radial direction and the $\mathrm{Z}$-axis to the axial direction of the artery. In all cases, the conditions are assigned such that rigid body motions are eliminated and the deformation gradient is homogeneous, by fixing plane $S 1, S 6$ and $S 5$ in the X, Y and Z-direction, respectively.

To reproduce pulmonary and aortic loading conditions, face $S 4$, corresponding to the inner surface of the artery, was loaded with a pressure $p_{i}$, with $i=P, A$ (for pulmonary and aortic pressure, respectively). Face $S 2$ was loaded with the corresponding hoop stress derived according to Laplace law $\sigma_{h}=\frac{p_{i} d_{i}}{2 t_{i}}$, where $d_{i}$ and $t_{i}$ are the diameter and wall thickness measured in the artery in pulmonary and aortic positions during the experiments described in section 2.3.1. The axial stretch was kept constant by fixing face $S 3$ in the Z-direction. Note that axial prestretch is implicitly imposed through deposition stretches. Table 2 shows the numerical values used for these boundary conditions, which correspond to 
values measured during the sheep experiments described in section 2.3.1, and in more detail in [10].

To perform a virtual planar biaxial test, first a release step is performed, in which faces $S 1, S 6$ and $S 5$ are kept fixed, but all boundary conditions on the other faces are removed. This models tissue is tissue excision from the body before in vitro biaxial testing. The resulting configuration is considered as the reference configuration for imposing biaxial loading, but all previous 'history', i.e. the stored variables containing information of the mass fractions and deformation history (as described in section 2.2 is transferred using the restart function of Abaqus with the keywords *import, update=yes, state=yes. Finally, faces $S 2$ and $S 3$ are pulled by an equal amount in the Xand Z- direction, respectively.

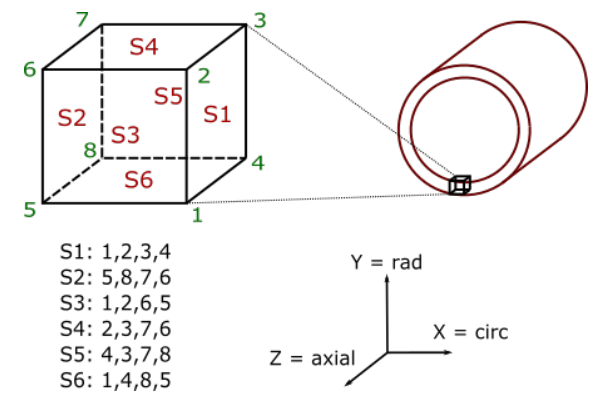

Figure 4: Schematic representation of the finite element used in our study and its orientation with respect to a complete artery. The nodes belonging to each face $(S 1-S 6)$ are listed for consistency with the text for assignment of boundary conditions.

After a virtual biaxial test, stress-strain curves are derived analogously to an in vitro test, i.e. by extracting the displacements of a marker on the sample $x_{M}, y_{M}$, and measuring the resulting forces acting on the pulled faces $R F_{x}$ and $R F_{y}$, throughout the test. Note that, since symmetry is applied along three faces, only one marker is needed (node 6 as indicated in Figure 4). The resulting stretch values are calculated as $\lambda_{i}=1+\frac{i_{M}}{l_{i 0}}$ for $i=x, y$. The resulting first Piola-Kirchhoff stress is calculated as $1 \mathrm{PK}_{i}=\frac{R F_{i}}{l_{i 0} t_{i 0}} \cdot l_{i 0}$ and $t_{i 0}$ are the length and thickness of the surface perpendicular to the $i$-th direction at the start of 
the biaxial test.

To evaluate the performance of the remodeling algorithm, four sets of virtual tests were performed, described below, and schematically represented in Figure [5.

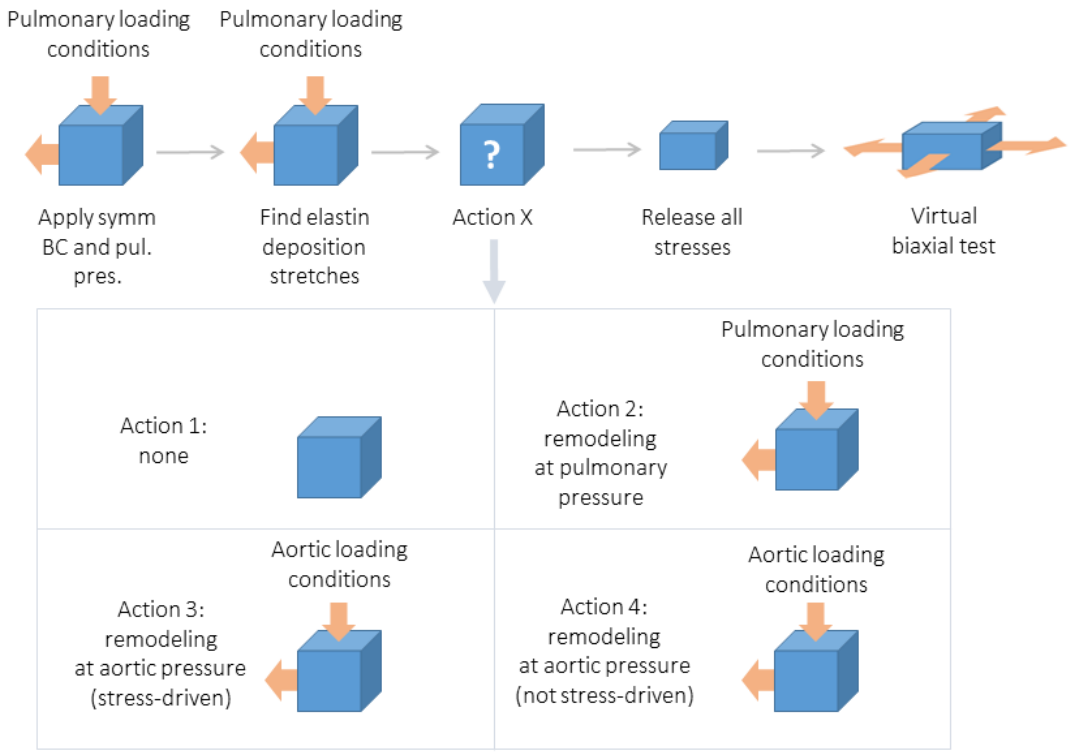

Figure 5: Schematic representation of the remodeling workflow with the four virtual tests described in the text.

Test $1\left(P_{p}^{\text {mod }}\right)$ : In the first test, pulmonary load is smoothly applied to the element in the first step. Next, while maintaining pulmonary loading conditions, elastin deposition stretches are assigned iteratively, where a time step corresponds to an iteration. After finding the deposition stretches, all loading conditions are released and a virtual biaxial test is carried out.

Test $2\left(P_{p r}^{m o d}\right)$ : In the second test, again pulmonary load is applied and deposition stretches are derived. Next, while still maintaining pulmonary 
loading conditions, the remodeling algorithm is applied for a total of 11 time steps, corresponding to 770 days. After this remodeling stage, all loading conditions are released and a virtual biaxial test is carried out.

Test $3\left(P_{a r_{-} \sigma}^{m o d}\right)$ : In the third test, pulmonary load is applied and deposition stretches are derived. Then the loading conditions are smoothly increased to reach the aortic level. At this load level, the remodeling algorithm is applied for 20 time steps, corresponding to 1400 days. After this remodeling stage, all loading conditions are released and a virtual biaxial test is carried out. Sensitivity to the $K_{\sigma}^{\text {coll }}$ rate parameter is investigated by repeating the simulations with three different $K_{\sigma}^{\text {coll }}$ values.

Test $4\left(P_{a r_{-} \text {noo }}^{\text {mod }}\right):$ In the final test, all steps are performed as in test 3 , but we remove the dependence of mass production and survival rates upon stressdifferences. Only the homeostatic constants of production and decay are used, meaning that $\Delta \sigma^{\alpha}$ and $\Delta \zeta^{\alpha}$ are assumed to be zero in equations 7 and 9 .

Table 1: Overview of the parameters used in the simulations.

\begin{tabular}{|c|c|c|c|c|c|}
\hline \multicolumn{2}{|c|}{ Material parameters } & \multicolumn{2}{|c|}{ Histological parameters } & \multicolumn{2}{|c|}{ Remodeling parameters } \\
\hline$C_{10}^{m}[\mathrm{MPa}]$ & $0.040 / 0.8$ & number of fiber families & 2 & $K_{q h}^{\text {elas }}\left[\right.$ days $\left.^{-1}\right]$ & 0 \\
\hline$k_{1}^{m}[\mathrm{MPa}]$ & $0.045 / 0.2$ & $\rho^{\operatorname{coll}_{i}}(0)[-]$ & 0.1 & $K_{q h}^{\text {coll }}\left[\right.$ days $\left.^{-1}\right]$ & $\log 2.0 / 70$ \\
\hline$k_{2}[-]$ & 2.94 & $\rho^{\text {elas }}(0)[-]$ & 0.8 & $K_{\sigma}^{\text {coll }}[-]$ & $\begin{array}{lll}1.0 & 2.0 & 10.0\end{array}$ \\
\hline$g_{a x}^{\text {elas }}[-]$ & 1.2 & $\kappa[-]$ & 0.10 & $m_{\max }[-]$ & 20.0 \\
\hline$g^{\text {coll }}[-]$ & 1.1 & $\alpha_{i}[\mathrm{rad}]$ & $\pm \frac{\pi}{8.0}$ & & \\
\hline
\end{tabular}


Table 2: Overview of the parameters used to apply pulmonary and aortic loading conditions in the simulations. These values correspond to those measured during the sheep experiments described in section 2.3.1 and in more detail in [10.

\begin{tabular}{cccccc}
\hline$p_{P}$ & $p_{A}$ & $d_{P}$ & $d_{A}$ & $t_{P}$ & $t_{A}$ \\
\hline $0.002 \mathrm{MPa}$ & $0.010 \mathrm{MPa}$ & $17.6 \mathrm{~mm}$ & $31.1 \mathrm{~mm}$ & $1.5 \mathrm{~mm}$ & $1.5 \mathrm{~mm}$ \\
\hline
\end{tabular}

\section{Results}

\subsection{Experimental results}

As described in section 2.3.1, planar biaxial tests were performed on healthy pulmonary arteries $\left(P_{p}^{e x p}\right)$, healthy aortas $\left(A_{a}^{\text {exp }}\right)$, and pulmonary autografts after six months of implantation in aortic position $\left(P_{a}^{e x p}\right)$. Figure 6 shows stressstretch curves of these tensile tests for two sheep, with two tests per sheep per condition. Figure 7 shows histological slices for each of the tissue types, and figure 8 shows an image of the actual autograft in aortic position immediately after surgical implantation. The complete set of results can be found in [10].

\subsection{Numerical results}

Using the workflow described in section 2.3.2 a 'virtual' simplified Ross procedure was simulated, after which virtual planar biaxial tests were carried out on healthy pulmonary artery $\left(P_{p}^{m o d}\right)$, pulmonary artery that was allowed to remodel under pulmonary pressure $\left(P_{p r}^{\text {mod }}\right)$ and pulmonary artery that remodeled under aortic loading conditions. The latter was performed first with stressdependent production and survival rates of collagen, and then with constant production and survival rates of collagen. Figure 9 shows stress-stretch curves of the four virtual biaxial tests. Note that, due to time discretization and with $\tau_{n}=10$, the time resolution is 70 days.

Figure 10 shows how the total collagen mass evolves during the remodeling stages of $P_{p r}^{m o d}, P_{a r_{-} \sigma}^{\text {mod }}$ and $P_{a r_{-} n o \sigma}^{m o d}$. For $P_{a r_{-} \sigma}^{\text {mod }}$, three different values of $K_{\sigma}^{\text {coll }}$ were used. Figure 11 shows the evolution of the average stress along a collagen 

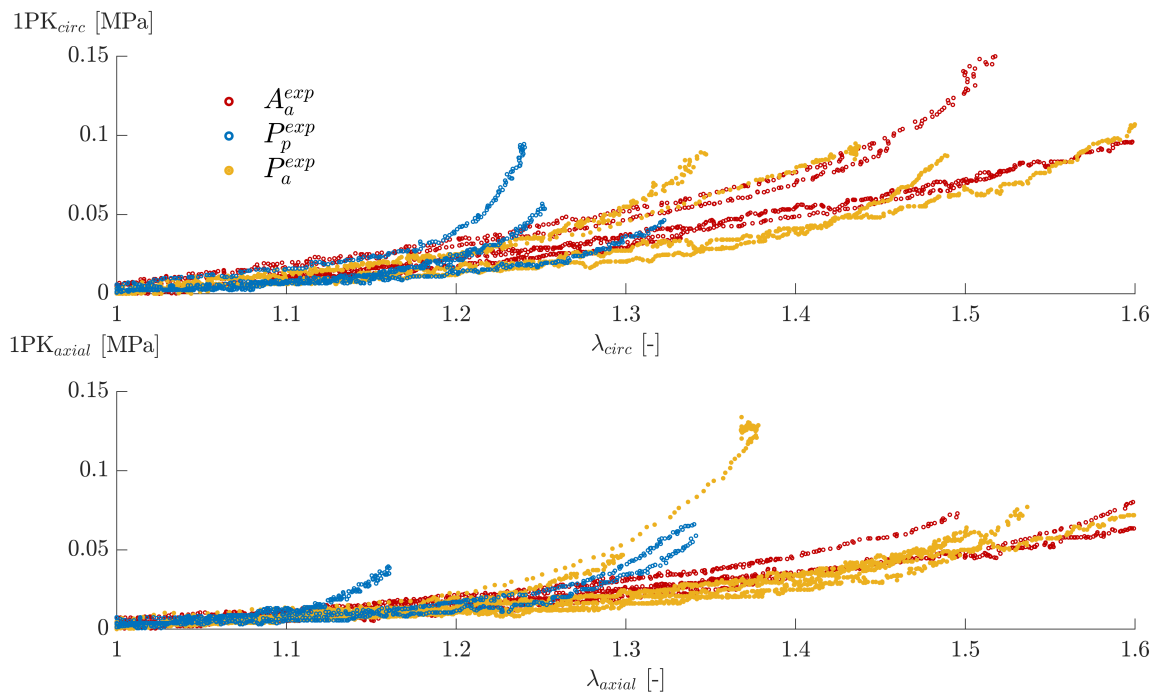

Figure 6: Stress-stretch curves of planar biaxial tests of healthy pulmonary artery $\left(P_{p}^{e x p}\right)$, pulmonary autograft after 6 months of implantation $\left(P_{a}^{e x p}\right)$, and healthy aortic tissue $\left(A_{a}^{e x p}\right)$ of two sheep. Per sheep, two tests per condition are shown. Further details can be found in [10].

fiber family for each of the aforementioned variations. These stress values are averaged out over the different cohorts of a family, weighted according to their current mass fraction.

The simulations were also repeated for varying levels of $\tau_{n}$, i.e. the amount of cohorts present within one fiber family, to investigate the effect of the discretization. Figure 12 shows how the steady state result is not affected by the level of $\tau_{n}$, for the ranges that were chosen here.

Figure 13 shows the evolution of the three principal Cauchy stress components in the elastin constituent, again for each of the aforementioned variations. Finally, Figure 14 shows the circumferential stretch throughout the different consecutive algorithm stages, for each of the aforementioned variations.

\subsection{Combined results}

As mentioned in the discussion, stiffer material parameters than the ones corresponding to the experimental data were used for reasons of numerical stability. 


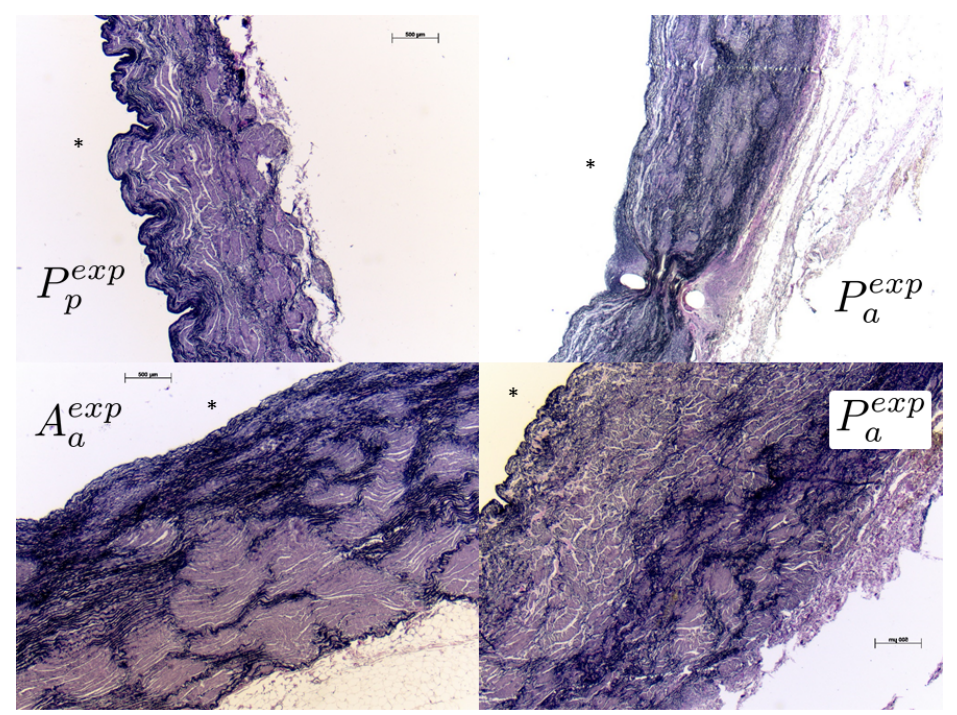

Figure 7: Histological slices of each tissue type (native pulmonary artery $P_{p}^{e x p}$, pulmonary autograft $P_{a}^{e x p}$, and native aortic tissue $A_{a}^{e x p}$ ) stained with Elastica van Gieson staining. Images are taken with a $25 \times$ magnification in a transversal plane, i.e. in a circumferential cross section. The scale bar indicates a distance of $500 \mu \mathrm{m}$, the asterisk $*$ indicates the luminal side. Two images of remodeled autograft are shown, taken from different locations on the same transversal plane, to indicate the inhomogeneous remodeling pattern. Further details can be found in 31 . 


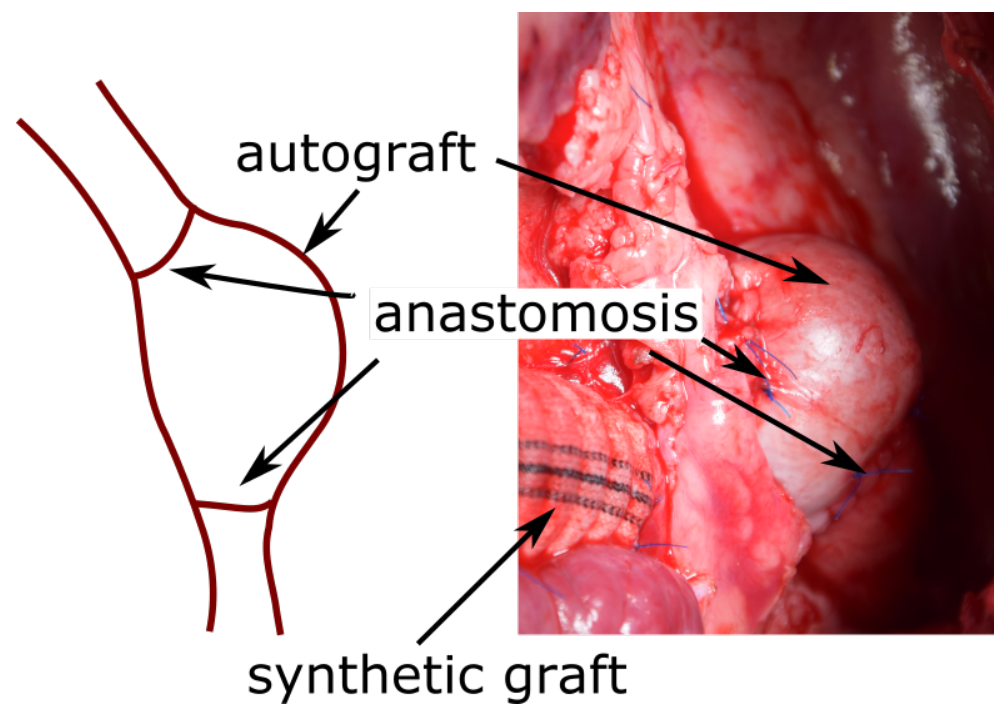

Figure 8: Image just after surgical implantation, showing the bulging pulmonary autograft in aortic position. The anastomoses are the locations where the autograft is sutured to the aorta. The synthetic graft that replaces the pulmonary artery is also visible at the bottom left of the picture.
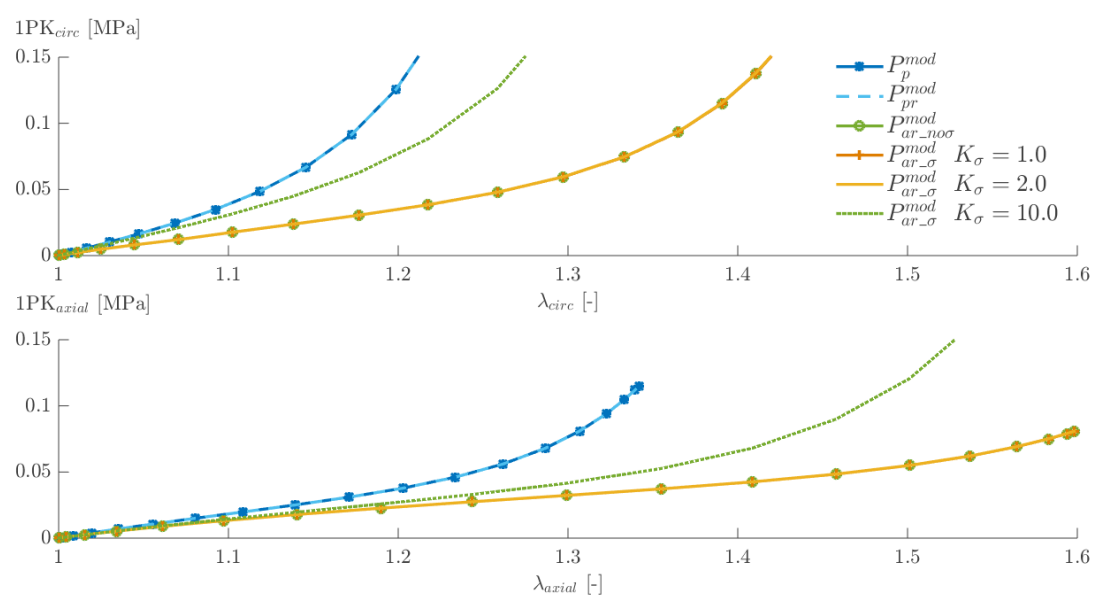

Figure 9: Stress-stretch curves of the virtual biaxial tests of healthy pulmonary artery $\left(P_{p}^{\text {mod }}\right)$, pulmonary artery that was allowed to remodel under pulmonary pressure $\left(P_{p r}^{m o d}\right)$, pulmonary artery that remodeled under aortic loading conditions with constant production and survival rates $\left(P_{a r_{-} \text {noo }}^{\text {mod }}\right)$ and pulmonary artery that remodeled under aortic loading conditions with stress-dependent production and survival rates $\left(P_{a r_{-} \sigma}^{m o d}\right)$. For the latter, three values of $K_{\sigma}^{\text {coll }}$ were tested. 


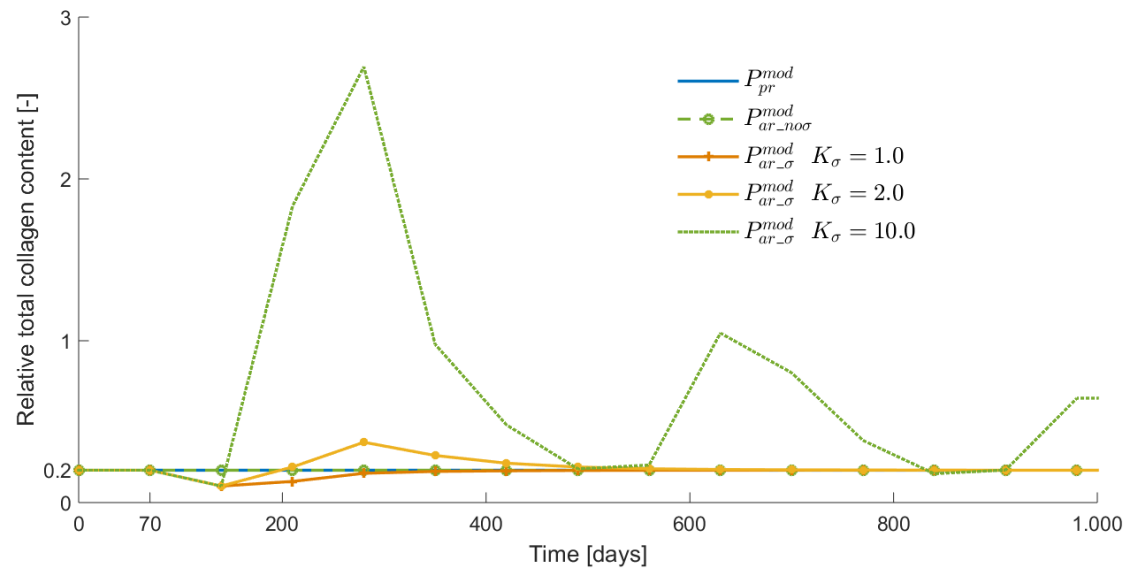

Figure 10: Evolution of the relative total collagen mass, i.e. $\frac{\rho^{\text {coll }}(s)}{\rho(0)}$, for each of the described variations. Time $=0$ is the time at which the autograft is placed under aortic loading conditions.

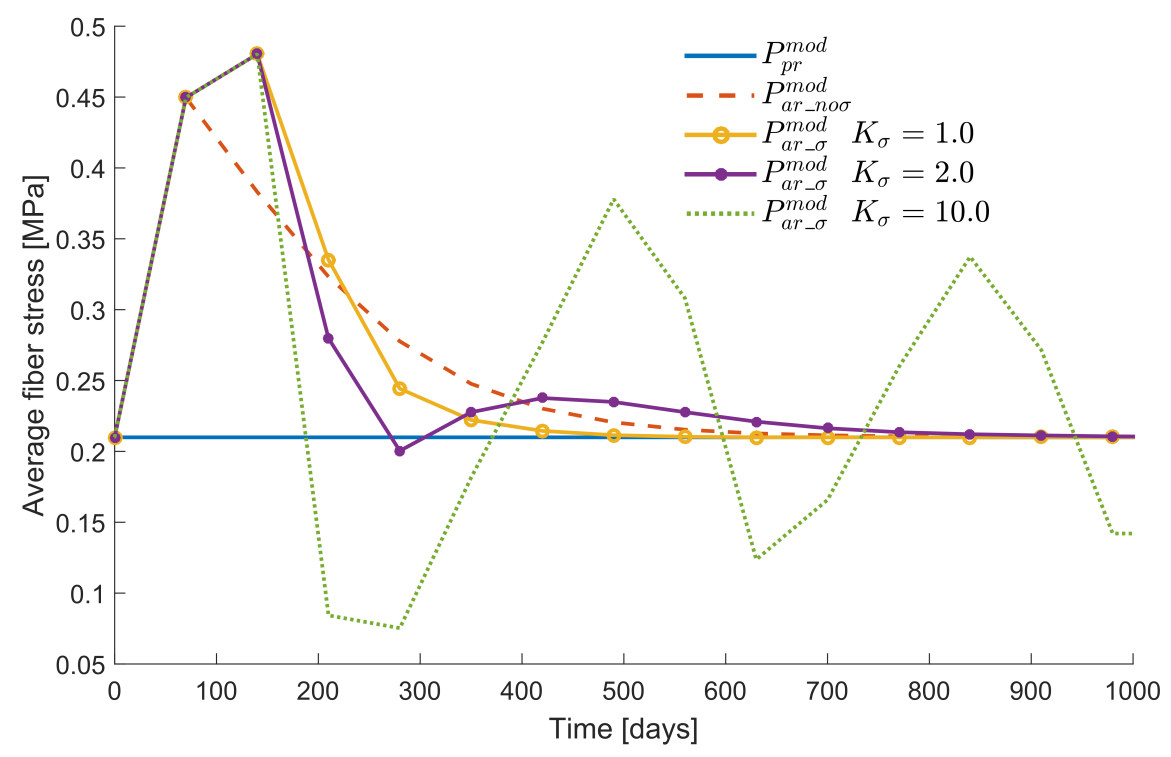

Figure 11: Evolution of the average fiber stress, as computed in equation 23 for the first collagen fiber family for each of the described variations. Time $=0$ is the time at which the autograft is placed under aortic loading conditions. 


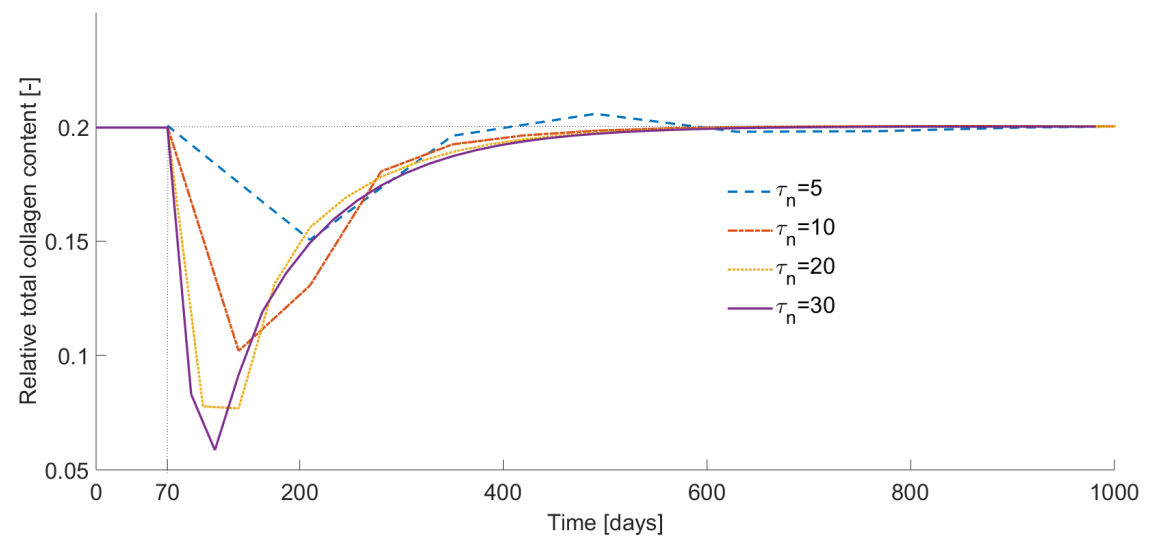

Figure 12: Evolution of the relative total collagen mass, i.e. $\frac{\rho^{\text {coll }}(s)}{\rho(0)}$, for varying levels of $\tau_{n}$, and with $K_{\sigma}^{\text {coll }}=1$. Time $=0$ is the time at which the autograft is placed under aortic loading conditions.
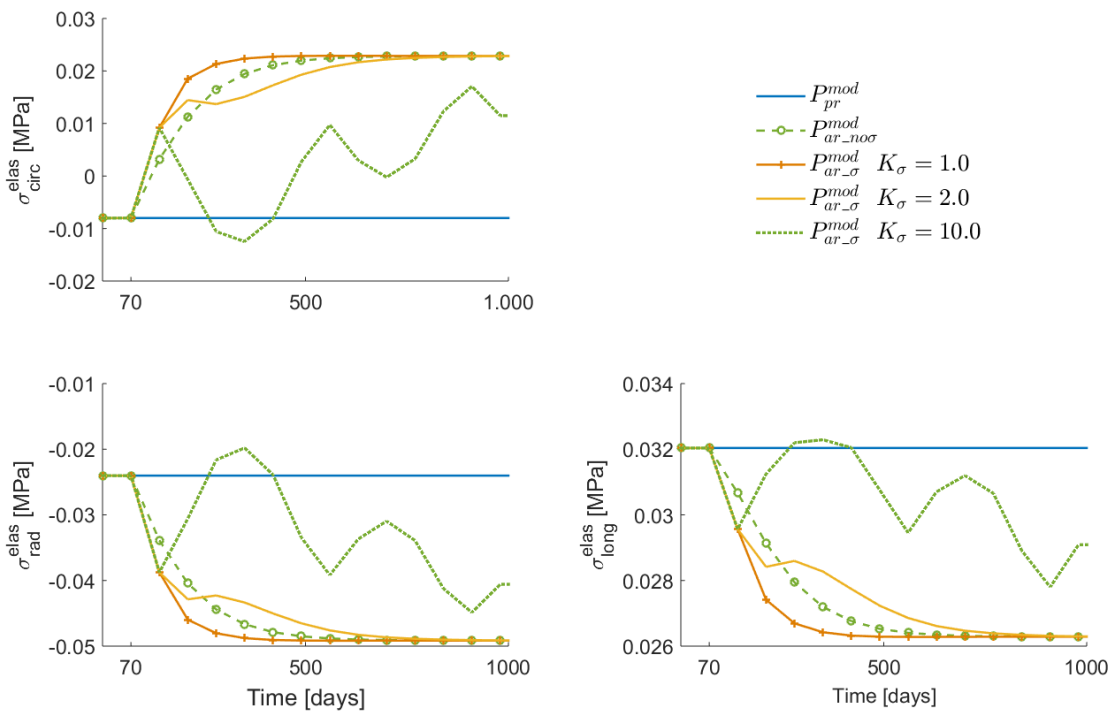

Figure 13: Evolution of the three principal Cauchy stress components in the elastin constituent for each of the described variations. Time $=0$ is the time at which the sample is placed under aortic loading conditions. 


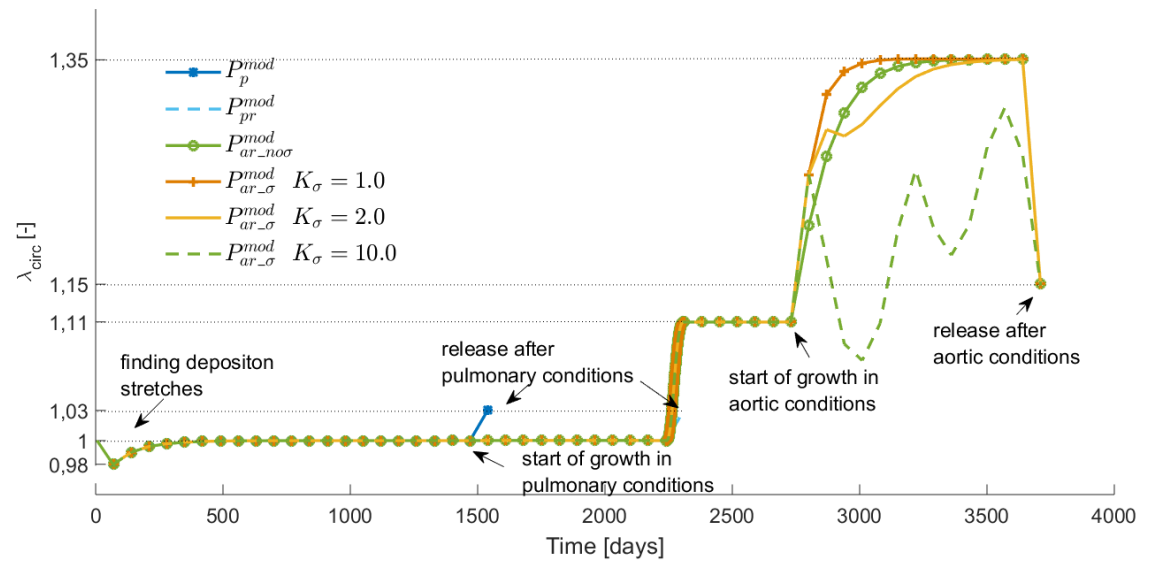

Figure 14: Evolution of the circumferential stretch throughout the different consecutive algorithm stages. Time $=0$ is the beginning of the simulation process, and time $=2730$ days is when the autograft is placed under aortic loading conditions.

However, to appreciate the qualitative agreement of the numerical remodeling results with the experimental observations, Figure 15 shows a combined version of figures 6 and 9 .

\section{Discussion}

\subsection{General observations}

In this paper, we performed numerical simulations of arterial remodeling using the constrained mixture theory. We qualitatively predicted the biomechanical response acquired by a pulmonary autograft after six months of implantation in aortic position.

Experimental observation (shown in Figure 6) reveals that the main evolution of the stress-stretch curve occurring in the pulmonary autograft from native state to remodeled state is a decrease of the initial slope combined with a delayed stiffening, indicating delayed recruitment of collagen fibers. The numerically obtained stress-stretch curves shown in Figure 9 imitate this behavior. The qualitative agreement between the experimental observation and the numerical implementation can also be appreciated in Figure 15, where the results 


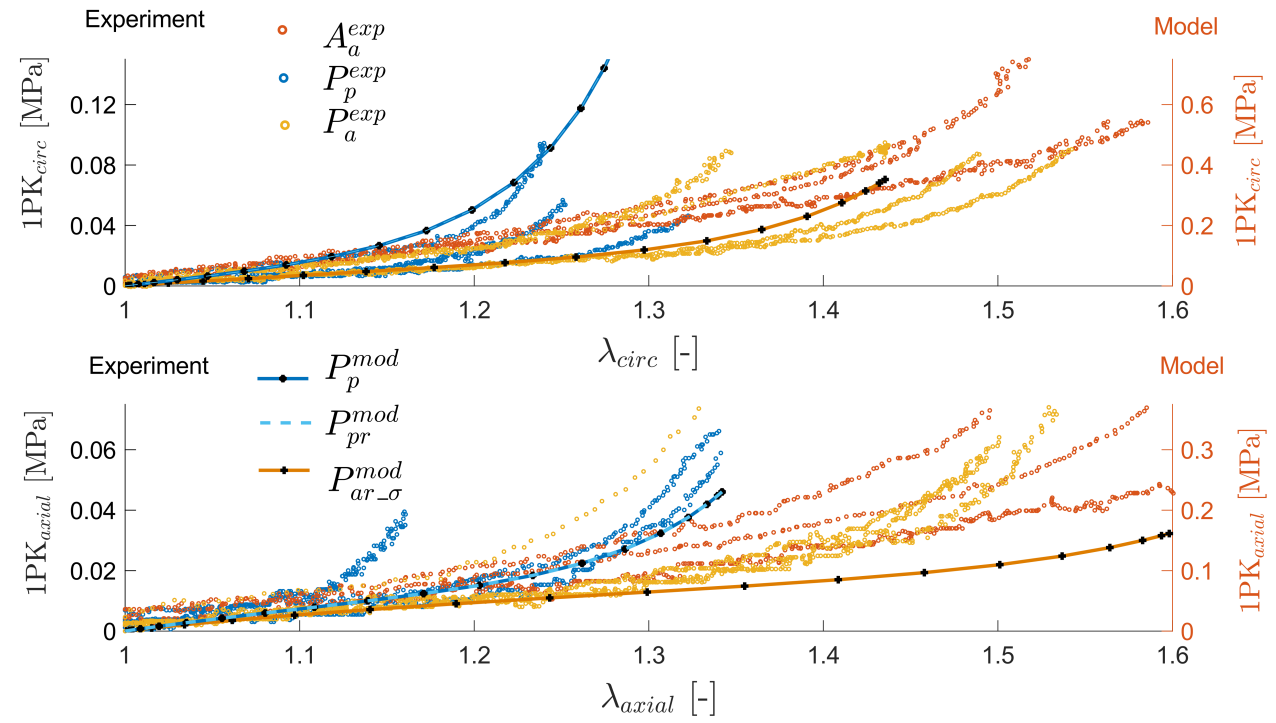

Figure 15: Scaled version of figures 6 and 9 through which the qualitative agreement between the experimental observations and numerical implementation can be appreciated. Note that different $y$-axes are used for the experimental and numerical results, since stiffer material parameters were used in the numerical case, for stability reasons. 
were scaled with respect to each other. Geometrically, the autograft develops a ballooned shape after implantation (see Figure 8), which is again numerically confirmed by the increased circumferential strain (Figure 13). The consistent way in which these features are accounted for by the model suggests that the assumptions of the model may be useful to understand better the remodeling process occurring in the pulmonary autograft.

Remodeling is accomplished by deposition of new collagen fibers and removal of existing ones, whereas native elastin fibers are preserved throughout the whole process. During the transient stage, the diameter of the autograft slightly increases each time a cohort of new collagen fibers is deposited, which is apparent by the increasing circumferential strain during the arterial growth stage in Figure 13. The diameter increases as the new cohort of collagen fibers takes a deposition stretch smaller than the stretch of all removed fibers, causing a decrease of the average stress taken by collagen fibers. The excessive stress is transferred to the elastin fibers. Elastin being more compliant, the transfer of stress to elastin induces an increase of strain, hence the gradual ballooning. This also explains the final biomechanical response with a delayed recruitment of collagen fibers.

Indeed, Figure 11 shows how the average stress in collagen fibers drastically increases when aortic conditions are initially imposed, but also how the average stress in collagen fibers recovers a stress state similar to the homeostatic stress (the same as in pulmonary conditions) after remodeling. Conversely, Figure 12 shows how the stress of elastin significantly increases to compensate for collagen loss of stress.

Elastin degradation was neglected in the algorithm, which can be justified by the relatively short time span of the experiment (6 months). Conversely, collagen was continuously removed and regenerated. Figure 10 shows how the total collagen mass density evolves. Despite the continuous turnover, the amount of collagen converges back to its initial quantity. The rate at which this convergence takes place depends on parameters of the algorithm: when remodeling is assumed stress-independent $\left(P_{a r_{-} \text {no }}^{\text {mod }}\right)$, the rate of deposition always equals the 
rate of degradation and the collagen content remains constant. When remodeling is stress-dependent $\left(P_{a r_{-} \sigma}^{\bmod }\right)$, initial perturbations in the amount of collagen can be observed, and the rate at which the collagen mass converges back to its initial amount is inversely dependent on the $K_{\sigma}^{c o l l}$ rate parameter.

Since the amount of collagen influences the overall stiffness of the material, the effect of the $K_{\sigma}^{\text {coll }}$ rate parameter can also be observed in Figures $9,11,12$ and 13. Note that the delayed convergence of collagen mass for a high value of $K_{\sigma}^{\text {coll }}$ results in a stiffer stress-strain behavior at the time step at which the virtual biaxial test was performed. This behavior oscillates until it converges to the behavior observed for the other variations.

The rate of convergence, or whether or not a similar type of oscillatory behavior might be present in reality cannot yet be verified through experimental data as longitudinal studies currently only report one time point after implantation. Hence, more extensive longitudinal studies are required to calibrate these parameters.

\subsection{Significance of the results}

The overall behavior predicted by the model is consistent with previous experimental observations of Schoof et al. [32, who showed that the developing pulmonary autograft in the aortic position retains its own typical pulmonary morphologic features and lacks major degenerative phenomena. They were also surprised to find that, by light microscopy in the autograft, the medial elastin lamellar pattern of the pulmonary wall had been preserved and that the autograft did not acquire the architecture of the aortic wall. Indeed, this was in contradiction with classical principles of remodeling that states that an artery would thicken in case of hypertension and that elastic arteries adapt in order to maintain the same stress in each lamellar unit of the media [1, 33]. Adaptation of the pulmonary wall to systemic hemodynamics is apparently accomplished in a different way as the number of lamellar units remains the same whereas the stress is multiplied by a factor 7. Schoof et al. 32 therefore concluded that the characteristic medial pattern of the pulmonary wall may be genetically 
predetermined and suggested that factors other than blood pressure contribute to determine vascular morphologic features.

Our simulations show that classical principles of homeostasis may still be satisfied for collagen in the autograft and the excessive stress would be taken by the elastin. Schoof et al. [32] also pointed out an acquired synthetic phenotype of smooth muscle cells (SMCs). This phenotype may be acquired due to the excessive deformations of the lamellar units. Consequently they speculate that SMCs lost their mechanosensitivity and the excessive stretch of elastin could not be sensed by them and could not be compensated by overproduction of extracellular matrix. Remodeling would be different if SMCs did not lose their mechanosensitivity. They would be able to signal the excess of stress, for instance to fibroblasts of the adventitia and to neighboring synthetic SMCs, and stimulate them to produce more extracellular matrix, hence thickening the artery and lessening the stress. This hypothesis is compatible with our results but should be investigated in more detail in future studies.

When studying the experiments reported in [31, histology on the remodelled autograft showed an inhomogeneous pattern across the circumference of the vessel. Some zones showed evident thickening with new matrix deposition, whereas other zones had no thickening at all. For instance, the top right image in Figure 7 shows no thickening with respect to the native pulmonary artery, whereas the bottom right image shows significant thickening with SMC hyperplasy. Both images were taken from a different section of the same transversal plane. A possible explanation for this inhomogeneous behavior may be found in studying Figures 3 and 8 . Due to the curved geometry of the pulmonary artery, the pulmonary autograft tends to bulge more on one side than on the other, inducing large strains in the bulging region, but rather moderate strains on the opposing side. We can hypothesize that in the overstretched region, remodeling occurs according to the mechanism we simulate here, i.e. with no volumetric growth as SMCs have lost their contractile phenotype and consequently have a reduced mechanosensitivity. Conversely, in the moderately stretched region, remodeling may occur through matrix deposition, although SMC hyperplasy is 
another phenomenon that is yet to be understood. Future experiments and simulations should be performed location-specifically to account for these regional variations.

\subsection{Limitations and future directions}

A number of simplifications and assumptions were made in the current study. First of all, simulations were performed on a single element on which boundary conditions of arteries were assigned using Laplace law. A more realistic cylindrical and multi-layered geometry would undoubtedly yield further insights.

Secondly, the material parameters used in the simulations were not the ones obtained from the actual pulmonary tissue of the sheep experiments, as the low experimental value of stiffness $C_{10}$ was the source of divergence in the solutions. It is a common issue for numerical implementations of Gasser-Ogden-Holzapfellike material models that convergence problems arise when the contribution of the matrix material is low compared to the contribution of the fibers. Further effort will focus on increasing the robustness of the model. Due to the divergence issues, we used larger values of stiffness $C_{10}$ that only provided a good qualitative agreement with experimental stress-stretch curves. Reaching quantitative agreement is the goal of our future efforts.

A trade-off for the number of cohorts within one collagen fiber family was set at $\tau_{n}=10$. This corresponds to a time step of 70 days, meaning that remodeling convergence is sought every 70 days. This is rather long, and might smooth out certain dynamic processes. Figure 12 shows how the relative total collagen content evolves for varying levels of $\tau_{n}=10$. If only interested in the steady state, a low value seems acceptable. However, if interested in effects at a smaller time scale during the remodeling period, a high value could be necessary. Memory restrictions will set a practical limit to this time scale, which is why a homogenized approach of the constrained mixture model [26] should be further explored.

Volumetric growth was only indirectly considered in our implementation. Hybrid elements were used to enforce incompressibility, but the density of the 
material was in fact allowed to change. This was done by calculating the current volumetric fractions of the material not by dividing by the current total density, but by the initial total density. Alternative ways exist to account for deposition of more material, where it is advised to enforce volumetric growth in the vessel's thickness direction, keeping the density constant [34, 35]. Future work will be directed at implementing the latter, since a more biofidelic representation of volumetric growth will become more important when modeling more realistic geometries.

Finally, as already mentioned, matrix deposition and SMC contractility were not taken into account in our current algorithm. This still permitted to obtain very promising results about autograft adaptation in its overstretched conditions. However, other possible applications should necessarily involve growth deformations which are common in many other situations of vascular adaptation.

\section{Conclusion}

In this paper, we proposed a remodeling algorithm based on the constrainedmixture theory, that simulates the continuous degradation and deposition of extracellular matrix in an artery. Using a discretization scheme in Abaqus with user-defined subroutines, the difference between ambient and homeostatic stress is calculated at every time step to mediate the turnover rate of a finite number of collagen fiber cohorts.

Promising results were obtained when predicting the adaptation of a pulmonary autograft over an extended period. They were compared against experimental data previously published on a follow-up study in sheep. The model faithfully reproduced the experimentaly observed dilatation and delayed stiffening in the stress-stretch behavior. Stress-mediated collagen production and removal influenced the global remodelling rate, but not the final tissue constitution and mechanical behaviour. In overstretched conditions, experimental results showed no volume change, justifying our modeling assumptions. Further 
development of growth modeling is however necessary to adequately capture adaptation in low stretch situations and other vascular specific situations.

Future work will also involve patient-specific modelling to assist clinicians in surgical planning, with the aim of reducing postoperative surgical complications, such as excessive vessel dilatation and accompanying valve regurgitation.

\section{Acknowledgements}

This work was supported by a postdoctoral fellowship and a travel grant from the Research Foundation Flanders (FWO - PDO12 to NF), a doctoral grant strategic basic research (SB 1S35316N to JV and SB 1 S56317N to EV), a KU Leuven category 2 research project (C2-ADAPT to FR) and an ERC consolidator grant (ERC-2014-CoG BIOLOCHANICS to SA). Finally, we would like to express our gratitude to Lisa De Backer and Gertjan Kloosterman for the helpful discussions imperative for successfully finishing this work.

\section{References}

[1] J. D. Humphrey, Cardiovascular solid mechanics: cells, tissues, and organs, Springer Science \& Business Media, 2013.

[2] J. C. Chambers, J. Somerville, S. Stone, D. N. Ross, Pulmonary autograft procedure for aortic valve disease, Circulation 96 (7) (1997) 2206-2214. arXiv:http://circ.ahajournals.org/content/96/ 7/2206, doi:10.1161/01.CIR.96.7.2206.

URL http://circ.ahajournals.org/content/96/7/2206

[3] T. E. David, A. Omran, J. Ivanov, S. Armstrong, M. P. de Sa, B. Sonnenberg, G. Webb, Dilation of the pulmonary autograft after the ross procedure., The Journal of thoracic and cardiovascular surgery 119 (2000) 210-220. doi:10.1016/S0022-5223(00)70175-9. 
[4] R. C. Elkins, M. M. Lane, C. McCue, K. E. Ward, Pulmonary autograft root replacement: mid-term results., The Journal of heart valve disease 8 (1999) 499-503; discussion 503-6.

[5] R. J. Smith, R. G. Bryant, Metal substitutions incarbonic anhydrase: a halide ion probe study., Biochemical and biophysical research communications 66 (1975) 1281-1286.

[6] W. P. Severin, Latex agglutination in the diagnosis of meningococcal meningitis., Journal of clinical pathology 25 (1972) 1079-1082.

[7] G. H. Gibbons, V. J. Dzau, The emerging concept of vascular remodeling., The New England journal of medicine 330 (1994) 1431-1438. doi:10. $1056 /$ NEJM199405193302008.

[8] A. Schmoldt, H. F. Benthe, G. Haberland, Digitoxin metabolism by rat liver microsomes., Biochemical pharmacology 24 (1975) 1639-1641.

[9] Y. Shi, J. E. O`Brien, J. D. Mannion, R. C. Morrison, W. Chung, A. Fard, A. Zalewski, Remodeling of autologous saphenous vein grafts : The role of perivascular myofibroblasts, Circulation 95 (12) (1997) 2684-2693. doi: $10.1161 / 01$. cir.95.12.2684.

[10] J. Vastmans, H. Fehervary, P. Verbrugghe, T. Verbelen, E. Vanderveken, J. V. Sloten, T. Treasure, F. Rega, N. Famaey, Biomechanical evaluation of a personalized external aortic root support applied in the ross procedure, Journal of the Mechanical Behavior of Biomedical Materials 78 (Supplement C) (2018) 164 - 174. doi:https://doi.org/10.1016/j. jmb.bm.2017.11.018.

URL http://www.sciencedirect.com/science/article/pii/ S1751616117305064

[11] A. Mookhoek, K. Krishnan, S. Chitsaz, H. Kuang, L. Ge, P. H. Schoof, A. J. J. C. Bogers, J. J. M. Takkenberg, E. E. Tseng, Biomechanics of Failed Pulmonary Autografts Compared to Native Aortic Roots, The Annals of 
Thoracic Surgerydoi:10.1016/j.athoracsur.2016.08.061.

URL http://dx.doi.org/10.1016/j.athoracsur.2016.08.061

[12] A. Mookhoek, K. Krishnan, S. Chitsaz, H. Kuang, L. Ge, P. H. Schoof, A. J. J. C. Bogers, J. J. M. Takkenberg, E. E. Tseng, Biomechanics of Failed Pulmonary Autografts Compared With Normal Pulmonary Roots, The Annals of Thoracic Surgery 102 (6) (2016) 1996-2002. doi:10.1016/ j.athoracsur.2016.05.010

URL http://dx.doi.org/10.1016/j.athoracsur.2016.05.010

[13] J. Humphrey, K. Rajagopal, A constrained mixture model for growth and remodeling of soft tissues, Mathematical Models and Methods in Applied Sciences 12 (03) (2002) 407-430. doi:10.1142/s0218202502001714.

[14] R. Skalak, Growth as a finite displacement field., in: R. S. D.E Carlson (Ed.), Proceedings of the IUTAM Symposium on Finite Elasticity, Martinus Nijhoff; The Hague, 1981, p. 347355.

[15] A. Tzeren, R. Skalak, Interaction of stress and growth in a fibrous tissue, Journal of Theoretical Biology 130 (3) (1988) 337-350. doi:10.1016/ s0022-5193(88) 80033-x.

[16] C. J. Cyron, J. D. Humphrey, Growth and remodeling of load-bearing biological soft tissues, Meccanica 52 (3) (2017) 645-664. doi:10.1007/ s11012-016-0472-5.

URL https://doi.org/10.1007/s11012-016-0472-5

[17] S. J. Mousavi, S. Avril, Patient-specific stress analyses in the ascending thoracic aorta using a finite-element implementation of the constrained mixture theory., Biomechanics and modeling in mechanobiology doi:10. 1007/s10237-017-0918-2.

[18] S. J. Mousavi, S. Farzaneh, S. Avril, Computational predict ions of damage propagation preceding dissection of ascending thoracic aortic aneurysms, International Journal for Numerical Methods in Biomedical Engineering. 
[19] C. A. Figueroa, S. Baek, C. A. Taylor, J. D. Humphrey, A computational framework for fluid-solid-growth modeling in cardiovascular simulations., Computer methods in applied mechanics and engineering 198 (2009) 35833602. doi:10.1016/j.cma.2008.09.013.

[20] A. Valentín, J. Humphrey, G. Holzapfel, A finite element-based constrained mixture implementation for arterial growth, remodeling, and adaptation: Theory and numerical verification, International Journal for Numerical Methods in Biomedical Engineering 29 (8) (2013) 822-849. doi:10.1002/cnm.2555 URL http://dx.doi.org/10.1002/cnm.2555

[21] S. Baek, K. R. Rajagopal, J. D. Humphrey, A theoretical model of enlarging intracranial fusiform aneurysms., Journal of biomechanical engineering 128 (2006) 142-149.

[22] P. N. Watton, N. A. Hill, M. Heil, A mathematical model for the growth of the abdominal aortic aneurysm., Biomechanics and modeling in mechanobiology 3 (2004) 98-113. doi:10.1007/s10237-004-0052-9.

[23] W. PN, V. Y, H. GA, Modelling Cerebral Aneurysm Evolution, Heidelberg: Springer-Verlag, 2011.

[24] S. Zeinali-Davarani, S. Baek, Medical image-based simulation of abdominal aortic aneurysm growth, Mechanics Research Communications 42 (2012) 107-117. doi:10.1016/j.mechrescom.2012.01.008.

[25] P. N. Watton, N. A. Hill, Evolving mechanical properties of a model of abdominal aortic aneurysm, Biomechanics and Modeling in Mechanobiology 8 (1) (2007) 25-42. doi:10.1007/s10237-007-0115-9.

[26] C. J. Cyron, R. C. Aydin, J. D. Humphrey, A homogenized constrained mixture (and mechanical analog) model for growth and remodeling of soft tissue, Biomechanics and Modeling in Mechanobiology 15 (6) (2016) 1389 1403. doi:10.1007/s10237-016-0770-9. 
[27] F. A. Braeu, A. Seitz, R. C. Aydin, C. J. Cyron, Homogenized constrained mixture models for anisotropic volumetric growth and remodeling, Biomechanics and Modeling in Mechanobiology 16 (3) (2016) 889-906. doi:10.1007/s10237-016-0859-1.

[28] T. C. Gasser, R. W. Ogden, G. A. Holzapfel, Hyperelastic modelling of arterial layers with distributed collagen fibre orientations., J R Soc Interface 3 (6) (2006) 15-35. doi:10.1098/rsif.2005.0073.

[29] C. Bellini, J. Ferruzzi, S. Roccabianca, E. DiMartino, J. Humphrey, A Microstructurally Motivated Model of Arterial Wall Mechanics with Mechanobiological Implications, Ann Biomed Eng 42 (3) (2014) 488-502. doi:10.1007/s10439-013-0928-x.A.

[30] D. Nolan, A. Gower, M. Destrade, R. Ogden, J. McGarry, A robust anisotropic hyperelastic formulation for the modelling of soft tissue, Journal of the Mechanical Behavior of Biomedical Materials 39 (Supplement C) (2014) 48 - 60. doi:https://doi.org/10.1016/j.jmbbm.2014. 06.016 .

URL http://www.sciencedirect.com/science/article/pii/ S1751616114001805

[31] E. Vanderveken, J. Vastmans, T. Verbelen, P. Verbrugghe, N. Famaey, E. Verbeken, T. Treasure, F. Rega, Reinforcing the pulmonary artery autograft in the aortic position with a textile mesh: a histological evaluation Interactive CardioVascular and Thoracic Surgery (2018) ivy134arXiv:/oup/backfile/content_public/ journal/icvts/pap/10.1093_icvts_ivy134/1/ivy134.pdf, doi:10.1093/icvts/ivy134. URL http://dx.doi.org/10.1093/icvts/ivy134

[32] P. H. Schoof, A. C. Gittenberger-De Groot, E. De Heer, J. A. Bruijn, M. G. Hazekamp, H. A. Huysmans, Remodeling of the porcine pulmonary auto- 
graft wall in the aortic position., The Journal of thoracic and cardiovascular surgery 120 (2000) 55-65. doi:10.1067/mtc.2000.106970.

[33] H. Wolinsky, S. Glagov, Structural basis for the static mechanical properties of the aortic media., Circ Res 14 (1964) 400-413.

[34] A. Grytsan, T. S. E. Eriksson, P. N. Watton, T. C. Gasser, Growth description for vessel wall adaptation: A thick-walled mixture model of abdominal aortic aneurysm evolution., Materials (Basel, Switzerland) 10. doi:10.3390/ma10090994.

[35] F. A. Braeu, A. Seitz, R. C. Aydin, C. J. Cyron, Homogenized constrained mixture models for anisotropic volumetric growth and remodeling., Biomechanics and modeling in mechanobiology 16 (2017) 889-906. doi:10.1007/s10237-016-0859-1. 